\title{
Prospect for dark matter annihilation signatures from gamma-ray observation of dwarf galaxies by LHAASO
}

\section{Xiao-Jun Bi ${ }^{*}$}

Key Laboratory of Particle Astrophysics, Institute of High Energy Physics, Chinese Academy of Sciences, Beijing 100049, China

E-mail: bixjeihep.ac.cn

\section{Su-Jie Lin}

Key Laboratory of Particle Astrophysics, Institute of High Energy Physics, Chinese Academy of Sciences, Beijing 100049, China

E-mail: Linsjeihep.ac.cn

\section{Peng-Fei Yin}

Key Laboratory of Particle Astrophysics, Institute of High Energy Physics, Chinese Academy of Sciences, Beijing 100049, China

E-mail: vinpfdihep.ac.cn

The Large High Altitude Air Shower Observatory (LHAASO) is a next generation observatory for high energy gamma-rays and cosmic rays with a wide field of view, which is sensitive to gamma-rays from $300 \mathrm{GeV}$ to $1 \mathrm{PeV}$. LHAASO is an ideal experiment to explore the gamma-ray signatures induced by annihilation of heavy dark matter (DM) particles in dwarf spheroidal satellite galaxies (dSphs). In this study, we investigate the LHAASO sensitivity to the DM annihilation at DM masses above $1 \mathrm{TeV}$. We consider nineteen $\mathrm{dSphs}$ with large $J$-factors and incorporate the statistical uncertainties of the $J$-factor in a combined analysis. Comparing with current limits, we find that LHAASO is sensitive to the annihilation signatures for DM masses from several TeV up to $100 \mathrm{TeV}$.

36th International Cosmic Ray Conference -ICRC2019-

July 24th - August 1st, 2019

Madison, WI, U.S.A.

\footnotetext{
*Speaker.
} 


\section{Introduction}

Dark matter particles may still annihilate with a moderate rate today and produce high energy standard model particles, which could lead to detectable signatures in astrophysical observations. Among these signatures, the gamma-ray photon is an ideal tool to explore the nature of DM. Dwarf spheroidal satellites (dSphs) are very promising to search for the gamma-ray signature from DM annihilation, because they are large galactic DM substructures with high DM densities. Since there is no significant gamma-ray emission from the astrophysical process in dSphs, it is expected that searching for the DM signature is almost background-free.

Many space-borne detectors and ground-based telescopes have searched for the gamma-ray signature from $\mathrm{DM}$ annihilation in a wide energy range from $\sim \mathscr{O}(0.1) \mathrm{GeV}$ to $\mathscr{O}(1) \mathrm{TeV}$. However, the current limits on the annihilation cross section for heavy DM particles above $\sim \mathrm{TeV}$ are weak due to the low statistic of photons at high energies.

The large high altitude air shower observatory (LHAASO) is a hybrid cosmic ray and gammaray observatory located at $4410 \mathrm{~m}$ above sea level near Daocheng, Sichuan province, China $\left(100^{\circ} .01 \mathrm{E}\right.$, $29^{\circ} .35 \mathrm{~N}$ ) [ [W, []]. It is composed of a square kilometer particle detector array, a water Cherenkov detector array (WCDA), a wide field Cherenkov telescope array, and a high threshold shower core detector array. The strong background rejection power $(\sim 1 \%)$ and large field of view (FOV) $(\sim 2$ sr) of LHAASO will benefit the very high energy gamma-ray observation of dSphs. Therefore, it is very promising for LHAASO to explore the DM annihilation signature and place stringent constraints on the properties of heavy DM particles. In this analysis we investigate the prospects for DM annihilation signatures from the gamma-ray observations by LHAASO. We consider nineteen dSphs with large $J$-factors and include the statistical uncertainties of the $J$-factor in a combined analysis .

\section{Analysis method}

The observed gamma-ray flux in a particular energy bin from the pair annihilation of DM in an astrophysical system is

$$
\Phi=\frac{1}{4 \pi} \frac{\langle\sigma v\rangle}{2 m_{\chi}^{2}} \int_{E_{\min }}^{E_{\max }} \frac{d N_{\gamma}}{d E_{\gamma}} d E_{\gamma} \times J
$$

where $m_{\chi}$ is the DM mass, $\langle\sigma v\rangle$ is the thermal average velocity-weighted DM annihilation cross section, and $d N_{\gamma} / d E_{\gamma}$ is the initial spectrum of photons induced by DM annihilation. In this analysis, we assume that the gamma-ray signature is from a certain annihilation channel and use the PPPC 4DM package to calculate $d N_{\gamma} / d E_{\gamma}$ [四, 团]. The $J$-factor is the integral of the DM density squared along the line of sight (1.o.s) within a solid angle of $\Delta \Omega=2 \pi\left(1-\cos \alpha_{\text {int }}\right)$.

The DM density profile and the $J$-factor of dSphs can be derived from the Jeans analysis by using the results of the kinematic observation of stellar velocities (see e.g. Refs. [[5, G, 四]). In this analysis, we use the mean values and statistical uncertainties of the $J$-factors of nineteen dSphs listed in in Table $\mathbb{U}$, which are provided by $[\mathbb{8}, \mathbf{Q}]$. These $J$-factors are calculated within an integration angle $\alpha_{\text {int }}$ of $0.5^{\circ}$, or the maximum angular radius $\theta_{\max }$ of the dSph which is determined by the outermost member star in the observation. In order to obtain a large signal-to-background ratio, we choose the $J$-factor within a smaller integration angle as $\alpha_{\text {int }}=\min \left\{\theta_{\max }, 0.5\right\}$. 
Table 1: The astrophysical properties of nineteen dSphs within the LHAASO FOV. The listed columns for each dSph are the name, right ascension (RA.), declination (DEC.), effective time ratio $\left(r_{\text {eff }}\right)$, maximum angular radius $\left(\theta_{\max }\right)$. The $J$-factor and $\theta_{\max }$ of the dSphs are taken from Ref. [《]]. For the four dSphs marked with asterisks whose $J$-factors are not given in Ref. [[]], the $J$-factors are taken from Ref. [Q] ].

\begin{tabular}{cccccc}
\hline \hline Source & $\begin{array}{c}\text { RA. } \\
(\mathrm{deg})\end{array}$ & $\begin{array}{c}\text { DEC. } \\
(\mathrm{deg})\end{array}$ & $\begin{array}{c}r_{\text {eff }} \\
(\text { year })\end{array}$ & $\begin{array}{c}\theta_{\max } \\
(\mathrm{deg})\end{array}$ & $\begin{array}{c}\log _{10} J_{\text {obs }} \\
\left(\mathrm{GeV}^{2} \mathrm{~cm}^{-5}\right)\end{array}$ \\
\hline Boötes I & 210.02 & 14.50 & 0.352 & 0.47 & $18.2 \pm 0.4$ \\
Canes Venatici I & 202.02 & 33.56 & 0.398 & 0.53 & $17.4 \pm 0.3$ \\
Canes Venatici II & 194.29 & 34.32 & 0.399 & 0.13 & $17.6 \pm 0.4$ \\
Coma Berenices & 186.74 & 23.90 & 0.377 & 0.31 & $19.0 \pm 0.4$ \\
Draco & 260.05 & 57.92 & 0.442 & 1.30 & $18.8 \pm 0.1$ \\
Draco II & 238.20 & 64.56 & 0.451 & - & $18.1 \pm 2.8$ \\
Hercules & 247.76 & 12.79 & 0.348 & 0.28 & $16.9 \pm 0.7$ \\
Leo I & 152.12 & 12.30 & 0.346 & 0.45 & $17.8 \pm 0.2$ \\
Leo II & 168.37 & 22.15 & 0.372 & 0.23 & $18.0 \pm 0.2$ \\
Leo IV & 173.23 & -0.54 & 0.303 & 0.16 & $16.3 \pm 1.4$ \\
Leo V & 172.79 & 2.22 & 0.314 & 0.07 & $16.4 \pm 0.9$ \\
Pisces II & 344.63 & 5.95 & 0.327 & - & $16.9 \pm 1.6$ \\
Segue 1 & 151.77 & 16.08 & 0.357 & 0.35 & $19.4 \pm 0.3$ \\
Sextans & 153.26 & -1.61 & 0.299 & 1.70 & $17.5 \pm 0.2$ \\
Triangulum II & 33.32 & 36.18 & 0.403 & - & $20.9 \pm 1.3$ \\
Ursa Major I $_{\text {Ursa Major II }}^{158.71}$ & 51.92 & 0.432 & 0.43 & $17.9 \pm 0.5$ \\
Ursa Minor & 132.87 & 63.13 & 0.449 & 0.53 & $19.4 \pm 0.4$ \\
Willman 1 & 227.28 & 67.23 & 0.455 & 1.37 & $18.9 \pm 0.2$ \\
\hline
\end{tabular}

We assume a series of mimic observations to investigate the LHAASO sensitivity to the gamma-ray signature from DM annihilation. The expected background number induced by cosmicray particles $B$ and signature number from DM annihilation $S$ in LHAASO are calculated. Assuming the null result of the DM signature in the mimic observation, we take a Poisson sampling around $B$ and randomly generate the mimic event count $N$. In each energy bin with $E_{\max } / E_{\min }=3$, the expected $B$ from the direction of a dSph is given by

$$
B=\int_{E_{\min }}^{E_{\max }} \int_{\Delta \Omega} \int_{0}^{T} \zeta_{c r} \cdot \Phi_{p}(E) \cdot A_{\mathrm{eff}}^{p}\left(E, \theta_{\mathrm{zen}}(t)\right) \cdot \varepsilon_{p}(E) d t d \Omega d E,
$$

where observational time $T$ is taken to be one year in this analysis, and $\Phi_{p}(E)$ is the primary proton flux described by a single power-law. An additional factor $\zeta_{c r}=1.1$ is introduced to include the contributions of heavier primary cosmic-ray particles. The integral is performed within a solid angle of $\Delta \Omega=2 \pi \times\left[1-\cos \left(\max \left\{\alpha_{\text {int }}, \theta_{c}\right\}\right)\right] . \theta_{c}$ is the angular resolution of LHAASO and varies with from $2^{\circ}$ to $0.1^{\circ}$ with the increased energy of the incoming photon. The effective area $A_{\text {eff }}^{p}$ depending on the energy and zenith angle and is taken from Ref. [ए]]. We also list the effective time ratio $r_{\text {eff }}$ of dSphs in Table $\square$ to show their visibility. It is defined as the fraction of observation time during which the zenith angle $\theta_{\text {zen }}$ is smaller than $60^{\circ}$.

The $\gamma / p$ discrimination is crucial for this analysis. The energy-dependent quality factor $Q \equiv$ $\varepsilon_{\gamma} / \sqrt{\varepsilon_{p}}$ for WCDA is estimated in Ref. [W]], where $\varepsilon_{\gamma}$ and $\varepsilon_{p}$ are survival ratios of gamma-rays and primary protons. It is shown that $\varepsilon_{p}$ above $0.6 \mathrm{TeV}$ varies from $0.04 \%$ to $0.11 \%$ for $\varepsilon_{\gamma} \sim 50 \%$. For a conservative analysis, we set $\varepsilon_{p} \sim 0.278 \%$ for $\varepsilon_{\gamma} \sim 40.13 \%$. 
A likelihood ratio test is performed to explore the possible excess in the mimic observation. We use the method in Refs. [미, [3] to take the statistical uncertainty of the $J$-factor into account. The likelihood in all energy bins for the $j$-th dSph is defined as

$$
\mathscr{L}_{j}=\prod_{i} \mathscr{L}_{i j}\left(S_{i j} \mid B_{i j}, N_{i j}\right) \times \frac{1}{\ln (10) J_{\mathrm{obs}, j} \sqrt{2 \pi} \sigma_{j}} \times e^{-\left[\log _{10}\left(\mathrm{~J}_{j}\right)-\log _{10}\left(\mathrm{~J}_{\mathrm{obs}, j}\right)\right]^{2} / 2 \sigma_{j}^{2}},
$$

where $i$ and $j$ represent the $i$-th energy bin and $j$-th $\mathrm{dSph}$, respectively, $\mathscr{L}(\mathbf{S} \mid \mathbf{B}, \mathbf{N})$ is taken to be the Poisson distribution, $\log _{10}\left(\mathrm{~J}_{\mathrm{obs}, j}\right)$ and $\sigma_{j}$ are the observed mean values and standard deviations of the $\mathrm{J}$ factor. For given $\langle\sigma v\rangle, \log _{10}\left(\mathrm{~J}_{j}\right)$ is adjusted to maximize $\mathscr{L}_{j}$.

For a 95\% C.L. upper limit on the DM signature flux $S_{95}$ in the mimic observation, we require that the log-likelihood with the DM contribution decreases by $2.71 / 2$ from its maximum. Then we can obtain the limit on the DM annihilation cross section $\langle\sigma v\rangle_{95}$ at 95\% C.L. for given $\langle\sigma v\rangle$. For the joint analysis with several dSphs, the procedure is similar by adopting a combined likelihood $\mathscr{L}^{\text {tot }}=\prod_{j} \mathscr{L}_{j}$.

\section{LHAASO Sensitivities}

In this analysis, we investigate the DM annihilation signatures from nineteen dSphs, which are located in the FOV of LHAASO with favored declination angles and have significant DM contents. Four more dSphs are considered (Draco II, Leo V, Pisces II and Willman 1), compared with the searches of HAWC [14]]. We investigate the sensitivities to the DM annihilation cross section for five annihilation channels, including $b \bar{b}, t \bar{t}, \mu^{+} \mu^{-}, \tau^{+} \tau^{-}$and $\mathrm{W}^{+} \mathrm{W}^{-}$. Both the individual sensitivities for each $\mathrm{dSph}$ and the combined sensitivity from a joint analysis for all the selected $\mathrm{dSphs}$ are calculated. As an example, we show the results for the $b \bar{b}$ annihilation channel from one mimic observation in Fig. $\mathbb{\text { W. }}$

We consider the statistical uncertainty of the $J$-factor in the analysis, which would loosen the expected sensitivity. Even so, the expected sensitivity of LHAASO is still better than the current constraints placed by HAWC by a factor of $2 \sim 5$. This improvement can be attributed to the larger effective area and better $\gamma / p$ discrimination of LHAASO. The area of WCDA, which is about 4.5 times larger than that of HAWC, would provide an improvement factor of $\sim 2.1$. In the researches of HAWC [ए5, [6] $\varepsilon_{p}$ is always larger than $1 \%$ around $1 \mathrm{TeV}$ for $\varepsilon_{\gamma} \sim 50 \%$, while $\varepsilon_{p}$ is take to be $\sim 0.278 \%$ in this analysis. This difference would provide provide another improvement factor of $\sim 2$. Note that $\varepsilon_{p}$ adopted here is more conservative than that provided by Ref. [四]. Furthermore, for the high-latitude bright sources such as Ursa Major II, LHAASO located at the latitude of $\sim 29^{\circ}$ would be more sensitive than HAWC. This would also improve the the LHAASO senstitivity.

Our results show that the combined sensitivity is dominated by Segue 1, Ursa Major II and Triangulum II. This is because that these three dSphs have large $J$-factors and favorable locations in the FOV of LHAASO. The sensitivities for other selected dSphs are much weaker and would not significantly affect the combined limit. Among the selected nineteen dSphs, Triangulum II has almost the largest $J$-factor and is located near the center of the FOV of LHAASO. But it does not totally dominated the combined sensitivity, since Triangulum II is an ultra-faint $\mathrm{dSph}$ and its $J$ factor has large statistical uncertainty. Therefore, the analysis taking the uncertainty of the $J$-factor 


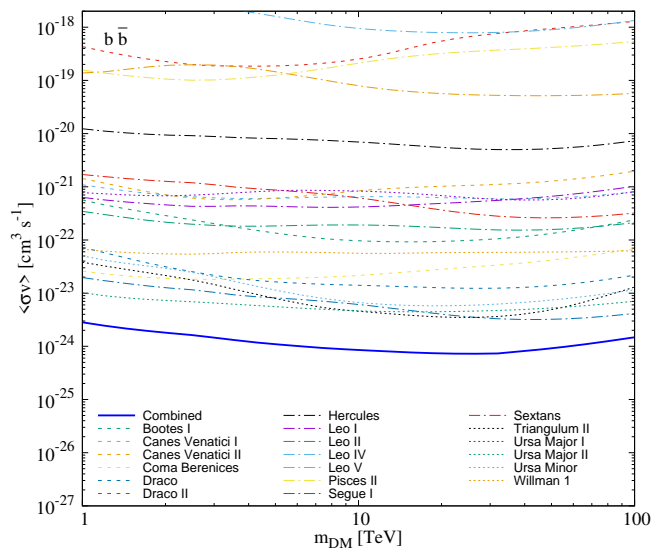

Figure 1: The individual expected sensitivities to the DM annihilation cross section $\langle\sigma v\rangle$ at $95 \%$ confidence level for nineteen $\mathrm{dSphs}$ of one year for the $\mathrm{b} \overline{\mathrm{b}}$ channel. The solid blue line represents the combined sensitivity.

into account is important and avoids the overestimation of the sensitivity for the dSph without enough kinematic data.

The photon number in the very high energy region is expected to be small, therefore the statistical fluctuation in the mimic observation should be taken into account. We perform 500 mimic observations under the null hypothesis. The median values and the two-sided $68 \%$ and $95 \%$ containment bands of the combined sensitivities for five DM annihilation channels are shown in Fig. $\mathbf{\square}$. The current constraints from five dSph observations, including the HAWC combined limit [U4]], Fermi-LAT combined dSph limit [ए]], HESS combined dSph limit [ए8], VERITAS Segue 1 limit [ㅁ] and MAGIC Segue 1 limit [ㅈ], are also shown for comparison.

The LHAASO sensitivity for the $\tau^{+} \tau^{-}$annihilation channel is the most strong among all the channels, because of the hard initial photon spectrum. It reaches $\sim 10^{-24} \mathrm{~cm}^{3} \mathrm{~s}^{-1}$ at DM masses above TeV. Compared with the current constraints placed by other experiments, LHAASO is also more sensitive above $\sim 2 \mathrm{TeV}, \sim 3 \mathrm{TeV}$ and $\sim 8 \mathrm{TeV}$ for the $\tau^{+} \tau^{-}, \mathrm{W}^{+} \mathrm{W}^{-}$and $b \bar{b}$ channels, respectively. For the $\mu^{+} \mu^{-}$and $t \bar{t}$ channels, LHAASO has a very strong capability to search for the annihilation signatures for $\mathrm{DM}$ masses from $\sim 1 \mathrm{TeV}$ to $100 \mathrm{TeV}$.

\section{Conclusion}

In this analysis, we investigate the sensitivity to the DM annihilation cross section for five DM annihilation channels by the LHAASO gamma-ray observation of dSphs. Our results show that the LHAASO combined sensitivity is dominated by three dSphs with large $J$-factors, including Segue 1, Ursa Major II and Triangulum II. Compared with the current constraints on the DM annihilation cross section, LHAASO is more sensitive at DM masses from several $\mathrm{TeV}$ up to $100 \mathrm{TeV}$.

\section{Acknowledgments}

We would like to thank Min Zha, Yan-Jin Wang, Yi-Qing Guo, Ying-Ying Guo, Han-Rong Wu and Zhi-Guo Yao for helpful and engaging discussions. This work is supported by the National Key 

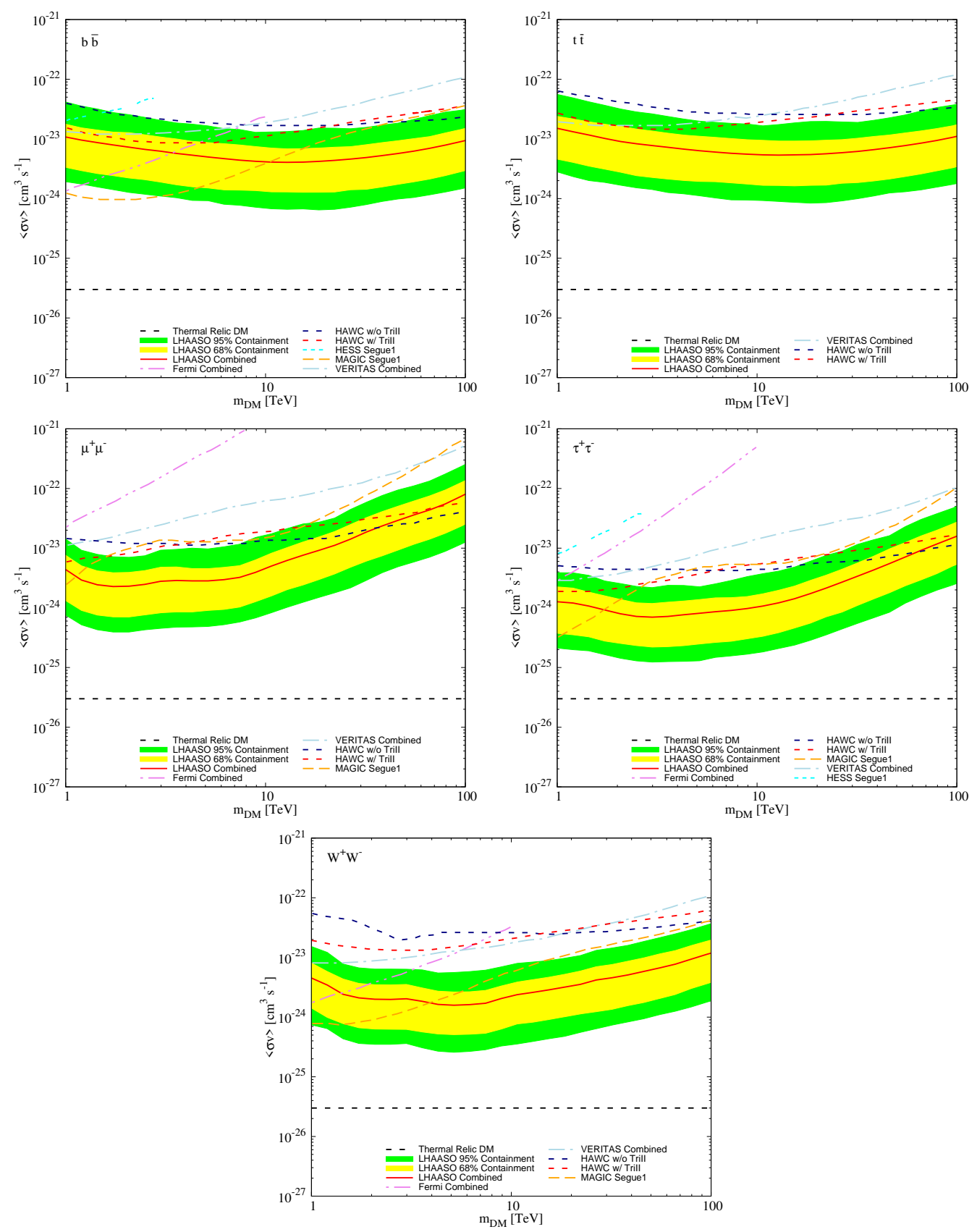

Figure 2: The LHAASO median combined sensitivities (red solid lines), and two-sided 68\% (yellow bands) and $95 \%$ (green bands) containment bands of one year for five annihilation channels, including $b \bar{b}, \mathrm{t} \bar{t}, \mu^{+} \mu^{-}$,

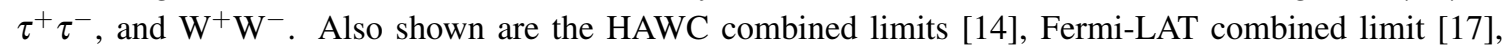

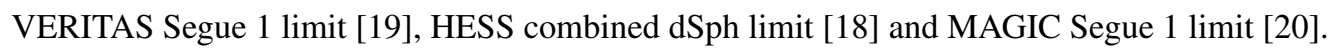


R \& D Program of China (Grant No. 2016YFA0400200), the National Natural Science Foundation of China (Grant Nos. U1738209, 11851303, 11835009), and the National Program for Support of Top-Notch Young Professionals.

\section{References}

[1] LHAASO collaboration, A future project at Tibet: The large high altitude air shower observatory (LHAASO), Chin. Phys. C 34(2010) 249.

[2] LHAASO collaboration, Status of LHAASO updates from ARGO-YBJ, Wucl.Instrum.Meth.A742 (2014)95.

[3] M. Cirelli, G. Corcella, A. Hektor, G. Hutsi, M. Kadastik, P. Panci et al., PPPC 4 DM ID: A Poor Particle Physicist Cookbook for Dark Matter Indirect Detection, एCAP 1103(2011) 05] [1012.4515].

[4] P. Ciafaloni, D. Comelli, A. Riotto, F. Sala, A. Strumia and A. Urbano, Weak Corrections are Relevant for Dark Matter Indirect Detection, VCAP1103 (2011)019] [1009.0224]].

[5] N. W. Evans, F. Ferrer and S. Sarkar, A 'Baedecker' for the dark matter annihilation signal, Phys. Rev. D69(2004)123501 astro-ph/0311145].

[6] L. E. Strigari, S. M. Koushiappas, J. S. Bullock, M. Kaplinghat, J. D. Simon, M. Geha et al., The Most Dark Matter Dominated Galaxies: Predicted Gamma-ray Signals from the Faintest Milky Way Dwarfs, Astrophys. J. 678(2008) 614 [0709.1510].

[7] G. D. Martinez, J. S. Bullock, M. Kaplinghat, L. E. Strigari and R. Trotta, Indirect Dark Matter Detection from Dwarf Satellites: Joint Expectations from Astrophysics and Supersymmetry, ECAP $0906(2009) 014$ [0902.4715].

[8] A. Geringer-Sameth, S. M. Koushiappas and M. Walker, Dwarf galaxy annihilation and decay emission profiles for dark matter experiments, Astrophys. J. 801 (2015) 74 [11408.0002].

[9] M. Hütten, C. Combet, G. Maier and D. Maurin, Dark matter substructure modelling and sensitivity of the Cherenkov Telescope Array to Galactic dark halos, VCAP 1609(2016) 047 [1606.04898].

[10] LHAASO collaboration, The Large High Altitude Air Shower Observatory (LHAASO) Science White Paper, 1905.02773 .

[11] LHAASO collaboration, Gamma/Proton separation study for the LHAASO-WCDA detector, DOS ICRC2017(2018) 842.

[12] FERMI-LAT collaboration, Searching for Dark Matter Annihilation from Milky Way Dwarf Spheroidal Galaxies with Six Years of Fermi Large Area Telescope Data, Phys. Rev. Lett. 115 (2015) ए31300] [प503.0264]].

[13] FERMI-LAT, DES collaboration, Searching for Dark Matter Annihilation in Recently Discovered Milky Way Satellites with Fermi-LAT, Astrophys. J. 834(2017)110 [1611. 03184 ].

[14] HAWC collaboration, Dark Matter Limits From Dwarf Spheroidal Galaxies with The HAWC Gamma-Ray Observatory, Astrophys. J. 853 (2018) 154 [15706.01277].

[15] HAWC collaboration, New method for gamma/hadron separation in hawc using neural networks, POSTCRC2015 (2016) 991 [15508.04370].

[16] A. U. Abeysekara et al., Observation of the crab nebula with the hawc gamma-ray observatory, Astrophys. J. 843(2017) 39 [1701.01778]. 
[17] FERMI-LAT collaboration, Dark matter constraints from observations of 25 Milky Way satellite galaxies with the Fermi Large Area Telescope, Phys. Rev. D 89(2014)042001] [11310.0828].

[18] H.E.S.S. collaboration, Search for dark matter annihilation signatures in H.E.S.S. observations of Dwarf Spheroidal Galaxies, Phys. Rev. D90(2014) 112012 [1410.2589].

[19] VERITAS collaboration, VERITAS Deep Observations of the Dwarf Spheroidal Galaxy Segue 1, Phys. Rev. D 85 (2012) 062001] [1202.2144]].

[20] MAGIC, FERMI-LAT collaboration, Limits to Dark Matter Annihilation Cross-Section from a Combined Analysis of MAGIC and Fermi-LAT Observations of Dwarf Satellite Galaxies, VCAP 1602 (2016)039 [1601.06590]. 